\title{
9q22 Deletion - First Familial Case
}

\author{
Linda Siggberg $^{1 * \dagger}$, Maarit Peippo ${ }^{2 \dagger}$, Marjatta Sipponen ${ }^{2}$, Taina Miikkulainen², Keiko Shimojima ${ }^{3}$, \\ Toshiyuki Yamamoto ${ }^{3}$, Jaakko Ignatius ${ }^{4}$ and Sakari Knuutila ${ }^{1}$
}

\begin{abstract}
Background: Only 29 cases of constitutional 9q22 deletions have been published and all have been sporadic. Most associate with Gorlin syndrome or nevoid basal cell carcinoma syndrome (NBCCS, MIM \#109400) due to haploinsufficiency of the PTCH1 gene (MIM *601309).

Methods and Results: We report two mentally retarded female siblings and their cognitively normal father, all carrying a similar $5.3 \mathrm{Mb}$ microdeletion at 9q22.2q22.32, detected by array CGH (244 K). The deletion does not involve the PTCH1 gene, but instead 30 other gene,s including the ROR2 gene (MIM *602337) which causing both brachydactyly type 1 (MIM \#113000) and Robinow syndrome (MIM \#268310), and the immunologically active SYK gene (MIM *600085). The deletion in the father was de novo and FISH analysis of blood lymphocytes did not suggest mosaicism. All three patients share similar mild dysmorphic features with downslanting palpebral fissures, narrow, high bridged nose with small nares, long, deeply grooved philtrum, ears with broad helix and uplifted lobuli, and small toenails. All have significant dysarthria and suffer from continuous middle ear and upper respiratory infections. The father also has a funnel chest and unilateral hypoplastic kidney but the daughters have no malformations.
\end{abstract}

Conclusions: This is the first report of a familial constitutional 9q22 deletion and the first deletion studied by arrayCGH which does not involve the PTCH1 gene. The phenotype and penetrance are variable and the deletion found in the cognitively normal normal father poses a challenge in genetic counseling.

\section{Background}

The first constitutional deletion of 9q22 was published in 1978 by Turleau [1] and since then only 29 patients have been reported, including two terminated fetuses. All have been sporadic (Table 1). In recent years, array comparative genomic hybridization (array CGH) has enabled more detailed reports on the genetic basis of 9q22 deletions. Reported deletion sizes vary from 2.3 $\mathrm{Mb}$ to more than $18 \mathrm{Mb}$ and no recurrent breakpoints have been observed (Table 1). While most reported deletions are sporadic, three balanced parental chromosomal rearrangements involving 9q have been detected and two of these have likely predisposed to the deletion in the descendants $[2,3]$ while one deletion lies outside the parental rearrangement [4]. Parental origin of the deleted chromosome has been traced in eleven cases of

\footnotetext{
* Correspondence: linda.siggberg@helsinki.fi

+ Contributed equally

'Department of Pathology, Haartman Institute and HUSLAB, University of Helsinki and Helsinki University Central Hospital, Haartmaninkatu 3, 00014 Helsinki, Finland

Full list of author information is available at the end of the article
}

which eight were paternal [2 (patient 3), 3 (patient 1 ), 5-8,] and three maternal [3 (patient 2), 9, 10] (Table 1). Characteristic to all these deletions are non-recurrent breakpoints leading to variable gene composition and an inconsistent phenotype. Most of them, however, span the PTCH1 gene (MIM *601309) and associate with Gorlin syndrome or nevoid basal cell carcinoma (NBCCS, MIM \#109400) due to haploinsufficiency of PTCH1.

We describe a family where two mentally retarded siblings and their cognitively normal father share an identical 5,3 Mb deletion at 9q22.2q22.32, not including PTCH1, and discuss genotype-phenotype correlation in these patients.

\section{Methods \\ Cytogenetic analysis}

Chromosome metaphase spreads of patients 1, 3, and the healthy mother were analyzed by standard G-banding karyotype analysis (400 band resolution). Additional subtelomere-fluoresence in situ hybridization (FISH) 
Table 1 Summary of clinical and molecular features of previously reported patients with constitutional 9 q22 deletions and the present ones.

\begin{tabular}{|c|c|c|c|c|c|c|c|c|c|c|c|c|c|}
\hline Reference & $\begin{array}{l}\text { Patient's } \\
\text { age and } \\
\text { gender }\end{array}$ & $\begin{array}{l}\text { Postnatal } \\
\text { height }\end{array}$ & $\begin{array}{l}\text { Postnatal } \\
\text { OFC }\end{array}$ & CNS features & Malformations & Dysmorphic features & $\begin{array}{l}\text { Clinical Gorlin } \\
\text { syndrome }\end{array}$ & $\begin{array}{l}\text { Method of } \\
\text { detection }\end{array}$ & $\begin{array}{l}\text { Locus of the } \\
\text { deletion }\end{array}$ & $\begin{array}{l}\text { Parental origin } \\
\text { and/or } \\
\text { Parental } \\
\text { chromosomal } \\
\text { rearrangement }\end{array}$ & $\begin{array}{l}\text { Size of } \\
\text { deletion }\end{array}$ & PTCH1 & ROR2 \\
\hline [28] & $\begin{array}{l}\begin{array}{l}1 y 11 \mathrm{~m} \\
\text { male }\end{array}\end{array}$ & $+3,8 \mathrm{SD}$ & $>p 90$ & $\begin{array}{l}\text { global delay } \\
\text { hypotonia } \\
\text { no falx calcification }\end{array}$ & $\begin{array}{l}\text { right hydronephrosis } \\
\text { left multicystic kidney } \\
\text { left hand preaxial } \\
\text { polydactyly }\end{array}$ & $\begin{array}{l}\text { hypertelorism } \\
\text { frontal bossing } \\
\text { epicanthi } \\
\text { low nasal bridge } \\
\text { low-set ears } \\
\text { auricular pits } \\
\text { long philtrum } \\
\text { high palate } \\
\text { short, webbed neck } \\
\text { sacral dimple }\end{array}$ & $\begin{array}{l}\text { with reservations to } \\
\text { young age }\end{array}$ & $\begin{array}{l}\text { aCGH (Agilent } \\
180 \text { chip) and } \\
\text { FISH }\end{array}$ & $\begin{array}{l}\text { 9q22.3 de } \\
\text { novo }\end{array}$ & & $2,44 \mathrm{Mb}$ & deleted & $\mathrm{nr}$ \\
\hline [14] & $\begin{array}{l}2 \text { y } 3 \mathrm{~m} \\
\text { male }\end{array}$ & > p97 & p75-97 & $\begin{array}{l}\text { moderate MR, } \\
\text { wide cranial sutures, } \\
\text { open posterior } \\
\text { fontanelle }\end{array}$ & $\begin{array}{l}\text { submucous cleft } \\
\text { palate, } \\
\text { pectus excavatum }\end{array}$ & $\begin{array}{l}\text { epicanthic folds, } \\
\text { wide and short neck, } \\
\text { low nuchal hairline, } \\
\text { wide nasal bridge, } \\
\text { low-set and posteriorly } \\
\text { rotated ears, } \\
\text { micrognathia, widely } \\
\text { spaced nipples, } \\
\text { small teeth, } \\
\text { deep plantar grooves }\end{array}$ & $\begin{array}{l}\text { with reservations to } \\
\text { young age }\end{array}$ & $\begin{array}{l}\text { aCGH at } 1 \mathrm{Mb} \\
\text { resolution }\end{array}$ & $\begin{array}{l}\text { 9q22.32q31.1 } \\
\text { de novo }\end{array}$ & & $\begin{array}{l}6,54-8,12 \\
\mathrm{Mb}\end{array}$ & deleted & $\mathrm{nr}$ \\
\hline [5] & $\begin{array}{l}\begin{array}{l}3 \text { y } 9 \mathrm{~m} \\
\text { female }\end{array}\end{array}$ & $+2,2 \mathrm{SD}$ & $+3,5 \mathrm{SD}$ & $\begin{array}{l}\text { normal development, } \\
\text { hypotonia, spasticity, }\end{array}$ & $\begin{array}{l}\text { cleft lip-palate, } \\
\text { pigmented cyst on } \\
\text { shoulder (ectopic } \\
\text { meninx) } \\
\text { ASD }\end{array}$ & $\begin{array}{l}\text { frontal bossing } \\
\text { epicanthic folds, broad } \\
\text { eyebrows, synophrys, } \\
\text { c-a-l spot on legs and arm }\end{array}$ & $\begin{array}{l}\text { yes } \\
\text { palmar \& plantar } \\
\text { pits }\end{array}$ & $\begin{array}{l}\text { aCGH (Agilent } \\
\text { 105A chip) }\end{array}$ & $\begin{array}{l}\text { 9q22.32 de } \\
\text { novo }\end{array}$ & paternal & $2.3 \mathrm{Mb}$ & deleted & $\mathrm{nr}$ \\
\hline [26, patient 1] & $12 \mathrm{y}$ male & $-1,1 \mathrm{SD}$ & $+1,7 \mathrm{SD}$ & $\begin{array}{l}\text { moderate } \mathrm{MR}, \\
\text { seizures/epilepsy }\end{array}$ & & $\begin{array}{l}\text { epicanthi } \\
\text { mouth small } \\
\text { upper lip thin } \\
\text { strabismus }\end{array}$ & $\begin{array}{l}\text { yes } \\
\text { falx calcification, } \\
\text { frontal } \\
\text { ganglioglioma, rib } \\
\text { anomalies, } \\
\text { odontogenic } \\
\text { ceratocysts, palmar } \\
\text { and plantar pits }\end{array}$ & $\begin{array}{l}\text { FISH with BAC } \\
\text { clones }\end{array}$ & $\begin{array}{l}\text { 9q21.33q22.33 } \\
\text { de novo }\end{array}$ & & $\begin{array}{l}15,33- \\
16,04 \mathrm{Mb}\end{array}$ & deleted & $\mathrm{nr}$ \\
\hline [26, patient 2] & 23 y male & $+1,9 \mathrm{SD}$ & $+2,6 \mathrm{SD}$ & $\begin{array}{l}\text { severe MR, seizures, } \\
\text { trigonocephaly, } \\
\text { craniosynostosis, }\end{array}$ & $\begin{array}{l}\text { cleft lip-palate } \\
\text { retinal detachement, } \\
\text { cataract, glaucoma } \\
\text { double urethra }\end{array}$ & hypertelorism & $\begin{array}{l}\text { yes } \\
\text { odonto-genic } \\
\text { ceratocysts, } \\
\text { thyroid } \\
\text { adenocarcinoma }\end{array}$ & $\begin{array}{l}\text { FISH with BAC } \\
\text { clones }\end{array}$ & $\begin{array}{l}\text { 9q21.33q31.1 } \\
\text { de novo }\end{array}$ & & $\begin{array}{l}18,08- \\
18,54 \mathrm{Mb}\end{array}$ & deleted & $\mathrm{nr}$ \\
\hline $\begin{array}{l}\text { [27, restudy } \\
\text { of patient } 1 \\
\text { originally } \\
\text { presented } \\
\text { in 33] }\end{array}$ & $\begin{array}{l}50 y \\
\text { female }\end{array}$ & normal & macrocephaly & $\begin{array}{l}\text { mild MR, } \\
\text { hypotonia, }\end{array}$ & $\begin{array}{l}\text { "rib and bone } \\
\text { anomalies" } \\
\text { kidney problems, one } \\
\text { eye blind }\end{array}$ & $\begin{array}{l}\text { frontal bossing, } \\
\text { palpebral fissures slant down, } \\
\text { epicanthi, } \\
\text { maxillary prognatism," } \\
\text { dense eyebrows, } \\
\text { dental anomalies, } \\
\text { delayed dental eruption }\end{array}$ & $\begin{array}{l}\text { yes } \\
\text { basocellular } \\
\text { carcinomas, } \\
\text { jaw cysts, } \\
\text { intracranial } \\
\text { calcification, } \\
\text { palmoplantar pits }\end{array}$ & $\begin{array}{l}\text { quantitative } \\
\text { multiplex } \\
\text { fluorescent PCR, } \\
\text { polymorphic } \\
\text { markers, long- } \\
\text { range PCR, } \\
\text { sequencing }\end{array}$ & $\begin{array}{l}\text { 9q22.32q22.33 } \\
\text { de novo }\end{array}$ & & $4,5 \mathrm{Mb}$ & deleted & $\mathrm{nr}$ \\
\hline
\end{tabular}


Table 1 Summary of clinical and molecular features of previously reported patients with constitutional 9q22 deletions and the present ones. (Continued)

\begin{tabular}{|c|c|c|c|c|c|c|c|c|c|c|c|c|c|}
\hline $\begin{array}{l}{[25, \text { patient }} \\
\text { G10] }\end{array}$ & 8 y male & $\mathrm{nr}$ & $\mathrm{nr}$ & $\begin{array}{l}\text { severe MR, } \\
\text { epilepsy, hydrocephalus }\end{array}$ & $\begin{array}{l}\text { inguinal hernia, } \\
\text { polydactyly } \\
\text { scoliosis }\end{array}$ & hypertelorism & $\begin{array}{l}\text { yes } \\
\text { palmar \& plantar } \\
\text { pits }\end{array}$ & HR microarray & $\begin{array}{l}\operatorname{inv}(9) \\
(\mathrm{q} 21.2 \mathrm{2q} 33.1)= \\
9 \mathrm{q} 22.32 \\
92,934,973 / \\
92,945,040- \\
98,137,216 / \\
98,141,889\end{array}$ & & $5 \mathrm{Mb}$ & deleted & $\mathrm{nr}$ \\
\hline $\begin{array}{l}\text { [16, patient G5, } \\
\text { restudy of } \\
\text { patient } \\
\text { originally } \\
\text { presented in 34] }\end{array}$ & 12 y male & $\mathrm{nr}$ & $>$ >p97 & $\begin{array}{l}\text { severe MR, epilepsy, } \\
\text { dilated lateral ventricles, } \\
\text { thin corpus callosum, } \\
\text { hydrocephalus }\end{array}$ & $\begin{array}{l}\text { hydronephrosis, } \\
\text { scoliosis }\end{array}$ & $\begin{array}{l}\text { hypertelorism } \\
\text { epicanthi } \\
\text { webbed neck }\end{array}$ & $\begin{array}{l}\text { yes } \\
\text { basal cell } \\
\text { carcinoma, } \\
\text { palmar and plantar } \\
\text { pits, } \\
\text { multiple jaw cysts } \\
\text { odonto-genic } \\
\text { ceratocysts, } \\
\text { calcification of falx } \\
\text { and tentorium } \\
\text { cerebellii }\end{array}$ & HR microarray & $\begin{array}{l}\text { 9q21.31q22.31 } \\
\text { de novo } \\
88,656,506 / \\
88,656,835- \\
99,686,554 / \\
99,687,352\end{array}$ & & $11 \mathrm{Mb}$ & deleted & deleted \\
\hline [17] & $\begin{array}{l}12 y \\
\text { female }\end{array}$ & $\mathrm{nr}$ & normal & $\begin{array}{l}\text { severe MR, brain } \\
\text { atrophy }\end{array}$ & $\begin{array}{l}\text { laryngeal stenosis, } \\
\text { pulmonary valve } \\
\text { stenosi, pectus } \\
\text { excavatum, } \\
\text { kyphoscoliosis, } \\
\text { hypoplastic clavicles }\end{array}$ & $\begin{array}{l}\text { down-slanting palpebral fissures, } \\
\text { epicanthi, prognatism, } \\
\text { asymmetric palpebral fissures, } \\
\text { broad eyebrows, } \\
\text { synophrys } \\
\text { high forehead, } \\
\text { pointed chin } \\
\text { short neck, } \\
\text { c-a-l-spots }\end{array}$ & yes & $\mathrm{aCGH}$ & $\begin{array}{l}\text { 9q22.1922.32 } \\
\text { de novo }\end{array}$ & & 7,7 Mb & deleted & deleted \\
\hline [6] & $\begin{array}{l}13 y \\
\text { female }\end{array}$ & $\mathrm{nr}$ & $\mathrm{nr}$ & $\begin{array}{l}\text { MR, } \\
\text { ventriculomegaly }\end{array}$ & mild macroglossia & $\begin{array}{l}\text { hypertelosim, } \\
\text { frontal bossing, } \\
\text { epicanthi, } \\
\text { ears posteriorly rotated, } \\
\text { teeth small, } \\
\text { prominent gingivae, } \\
\text { toenails hypoplastic, } \\
\text { mild hemihypertrophy }\end{array}$ & $\begin{array}{l}\text { yes } \\
\text { mandibular cysts, } \\
\text { plantar and palmar } \\
\text { spots, } \\
\text { rhabdomyosarcoma, } \\
\text { Wilms tumor }\end{array}$ & $\begin{array}{l}\text { karyotype, } \\
\text { polymorphic } \\
\text { markers at the } \\
\text { PTCH1 region }\end{array}$ & $\begin{array}{l}\text { 9q22q32 de } \\
\text { novo }\end{array}$ & paternal & $\mathrm{nr}$ & deleted & $\mathrm{nr}$ \\
\hline [7, patient 1] & 5 y male & $+2,5 \mathrm{SD}$ & $+2 \mathrm{SD}$ & severe MR & $\begin{array}{l}\text { umbilical hernia, } \\
\text { pectus excavatum, } \\
\text { trigonocephaly- } \\
\text { craniosynostosis }\end{array}$ & $\begin{array}{l}\text { epicanthi } \\
\text { mouth small } \\
\text { upper lip thin } \\
\text { ear pits } \\
\text { ears low set } \\
\text { ear lobules uplifted hyperlaxity, } \\
\text { short neck, }\end{array}$ & $\begin{array}{l}\text { with reservations to } \\
\text { young age }\end{array}$ & $\begin{array}{l}\text { aCGH at } 1 \mathrm{Mb} \\
\text { resolution }\end{array}$ & $\begin{array}{l}\text { 9q22.32q22.33 } \\
\text { de novo }\end{array}$ & paternal & $<6,5 \mathrm{Mb}$ & $\begin{array}{l}\text { not } \\
\text { tested }\end{array}$ & $\mathrm{nr}$ \\
\hline [7, patient 2] & $\begin{array}{l}8 y \\
\text { female }\end{array}$ & $+2 \mathrm{SD}$ & $>+3 S \mathrm{D}$ & $\begin{array}{l}\text { severe MR, seizures } \\
\text { trigonocephaly } \\
\text { ventriculomegaly } \\
\text { thin corpus callosum }\end{array}$ & $\begin{array}{l}\text { thyroglossal cyst with } \\
\text { sternal fistula, } \\
\text { no dentition, } \\
\text { umbilical hernia, } \\
\text { pectus excavatum, } \\
\text { kyphosis }\end{array}$ & $\begin{array}{l}\text { epicanthi, } \\
\text { palpebral fissures slant down, } \\
\text { small mouth, } \\
\text { thin upper lip, } \\
\text { thick ears } \\
\text { indentation of ear lobules. } \\
\text { short neck }\end{array}$ & $\begin{array}{l}\text { with reservations to } \\
\text { young age }\end{array}$ & $\begin{array}{l}\text { aCGH at } 1 \mathrm{Mb} \\
\text { resolution }\end{array}$ & $\begin{array}{l}\text { 9q22.32q22.33 } \\
\text { de novo }\end{array}$ & paternal & $<6,5 \mathrm{Mb}$ & $\begin{array}{l}\text { not } \\
\text { tested }\end{array}$ & $\mathrm{nr}$ \\
\hline
\end{tabular}


Table 1 Summary of clinical and molecular features of previously reported patients with constitutional 9q22 deletions and the present ones. (Continued)

\begin{tabular}{|c|c|c|c|c|c|c|c|c|c|c|c|c|c|}
\hline [27] & $5 \mathrm{~m}$ male & p75-90 & p90-97 & $\begin{array}{l}\text { MR, hypotonia } \\
\text { seizures } \\
\text { hydfrocephalus caused } \\
\text { by compression by } \\
\text { cerebellar vermis }\end{array}$ & $\begin{array}{l}\text { inguinal hernias, } \\
\text { PDA, } \\
\text { undescended testes, } \\
\text { high arched palate, } \\
\text { postaxial polydactyly } \\
\text { of left foot, } \\
\text { cervical ribs }\end{array}$ & $\begin{array}{l}\text { hypertelorism, prognatism, } \\
\text { broad face and } \\
\text { forehead, broad } \\
\text { nasal bridge, } \\
\text { supraorbital ridges well developed }\end{array}$ & yes & $\begin{array}{l}\text { HR karyotype, } \\
\text { aCGH, FISH }\end{array}$ & $\begin{array}{l}\text { 9q22.32q31.3 } \\
\text { de novo }\end{array}$ & paternal & $12 \mathrm{Mb}$ & deleted & $\mathrm{nr}$ \\
\hline [15] & 21 y male & $+0,2 \mathrm{SD}$ & $-1 S D$ & mild MR & $\begin{array}{l}\text { kyphosis, postaxial } \\
\text { polydactyly, } \\
\text { mild pulmonary valve } \\
\text { stenosis, inguinal } \\
\text { hernias, undescended } \\
\text { testes, hypodontia of } \\
\text { permanent teeth, } \\
\text { palate high arched, } \\
\text { uvula bifid, bilateral } \\
\text { nasal stenosis, } \\
\text { taurodonty of } 2^{\text {nd }} \\
\text { molars }\end{array}$ & $\begin{array}{l}\text { frontal bossing } \\
\text { epicanthi, } \\
\text { palperbral fissures slant down, } \\
\text { prognatism, synophrys, hypotelorism, } \\
\text { excess nuchal skin, } \\
\text { ears low-set and posteriorly rotated, } \\
\text { nares anteverted, } \\
\text { lips thick, } \\
\text { face high }\end{array}$ & $\begin{array}{l}\text { yes } \\
\text { calcification of cella } \\
\text { turgica and falx } \\
\text { cerebri, basal cell } \\
\text { nevus carcinomas, } \\
\text { jaw cysts }\end{array}$ & $\begin{array}{l}\text { HR karyotype, } \\
\text { FISH with BAC } \\
\text { clones }\end{array}$ & $\begin{array}{l}\text { 9q21.3931 de } \\
\text { novo }\end{array}$ & & $\begin{array}{l}15.3-15.6 \\
M B\end{array}$ & deleted & deleted \\
\hline [32] & $\begin{array}{l}29 y \\
\text { female }\end{array}$ & short & $\mathrm{nr}$ & & $\begin{array}{l}\text { poor vision, } \\
\text { telangiectatic nodule } \\
\text { on skin } \\
\text { hemivertebra T5, } \\
\text { scoliosis, } \\
\text { elongated clavicle }\end{array}$ & $\begin{array}{l}\text { hypotelorism, } \\
\text { ulnar deviation of hands }\end{array}$ & $\begin{array}{l}\text { yes } \\
\text { multiple basaliomas, } \\
\text { calcification of falx, } \\
\text { tentorium cerebri } \\
\text { and cella turcica, } \\
\text { mandibular cysts }\end{array}$ & karyotype & $9 \mathrm{q} 22.1 \mathrm{q} 31.2$ & $\begin{array}{l}\text { parents not } \\
\text { available }\end{array}$ & $\mathrm{nr}$ & $\begin{array}{l}\text { assumed } \\
\text { deleted }\end{array}$ & $\mathrm{nr}$ \\
\hline [4] & $\begin{array}{l}12 y \\
\text { female }\end{array}$ & $\mathrm{nr}$ & $\mathrm{nr}$ & $\begin{array}{l}\text { mild MR, } \\
\text { bridging of cella turcica, } \\
\text { broad cavum septi } \\
\text { pellucid, dilated cerebral } \\
\text { ventricles }\end{array}$ & $\begin{array}{l}\text { hyperopia, } \\
\text { deverticulum of the } \\
\text { renal calyx, occult } \\
\text { spina bifida L5-S4, } \\
\text { pectus excavatum, } \\
\text { bilateral patellar } \\
\text { dysplasia, unsual } \\
\text { clavicles, exostosis of } \\
\text { distal phalanx of } \\
\text { thumb } \\
\text { thumbs, abnormal } \\
\text { hypoplasia of maxilla }\end{array}$ & $\begin{array}{l}\text { hypertelorism, biparietal bossing, } \\
\text { epicanthi, palpebral fissures slant down, } \\
\text { prognatism, synophrys, } \\
\text { webbed neck, } \\
\text { synophrys and broad eyebrows, } \\
\text { low midface, } \\
\text { broad nose tip, } \\
\text { low set, posteriorly rotated ears }\end{array}$ & $\begin{array}{l}\text { yes } \\
\text { basalioma } \\
\text { basal cell nevi } \\
\text { trichoepithelioma }\end{array}$ & $\begin{array}{l}\text { HR karyotype, } \\
\text { FISH }\end{array}$ & $\begin{array}{l}\text { 9q22.32933.2 } \\
\text { de novo } \\
\text { outside the } \\
\text { maternal } \\
\text { translocation }\end{array}$ & $\begin{array}{l}\text { familial } t(9 ; 17) \\
\text { (q34.1p11.2)mat }\end{array}$ & $\mathrm{nr}$ & deleted & $\mathrm{nr}$ \\
\hline [30, patient A] & $\begin{array}{l}\text { age } \mathrm{nr}_{\text {, }} \\
\text { female }\end{array}$ & $\mathrm{nr}$ & $\mathrm{nr}$ & MR & & & $\mathrm{nr}$ & $\begin{array}{l}\text { karyotype, } \\
\text { aCGH }\end{array}$ & 9q21q22.1 & & $\mathrm{nr}$ & $\mathrm{nr}$ & deleted \\
\hline [30, patient B] & $\begin{array}{l}\text { age } \mathrm{nr}, \\
\text { female }\end{array}$ & $\mathrm{nr}$ & $\mathrm{nr}$ & MR & & & $\mathrm{nr}$ & $\begin{array}{l}\text { karyotype, } \\
\text { aCGH }\end{array}$ & $9 \mathrm{q} 22.1 \mathrm{q} 31.2$ & & $\mathrm{nr}$ & $\mathrm{nr}$ & deleted \\
\hline [8] & 6 y male & p50 & >p97 & $\begin{array}{l}\text { severe MR } \\
\text { hypotony }\end{array}$ & $\begin{array}{l}\text { PDA, severe scoliosis, } \\
\text { fingers slender, } 5^{\text {th }} \\
\text { finger camptodactyly, } \\
\text { palate high arched, } \\
\text { short metacarpals and } \\
\text { distal phalanges }\end{array}$ & $\begin{array}{l}\text { frontal bossing, } \\
\text { epicanthi, } \\
\text { palpebral fissures slant down, } \\
\text { ears low-set, } \\
\text { hypoplastic nostrils, } \\
\text { micrognarhia, } \\
\text { small nails }\end{array}$ & $\begin{array}{l}\text { with reservations to } \\
\text { young age } \\
\text { increasing nr of } \\
\text { nevi, } \\
\text { sole pits }\end{array}$ & $\begin{array}{l}\text { HR karyotype, } \\
\text { FISH, } \\
\text { genotyping }\end{array}$ & $\begin{array}{l}\text { 9q22.31q31.2 } \\
\text { de novo }\end{array}$ & paternal & $\begin{array}{l}\text { D95303 } \\
->\text { D95930 }\end{array}$ & deleted & deleted \\
\hline
\end{tabular}


Table 1 Summary of clinical and molecular features of previously reported patients with constitutional 9q22 deletions and the present ones. (Continued)

\begin{tabular}{|c|c|c|c|c|c|c|c|c|c|c|c|c|c|}
\hline$[3$, patient 1] & $\begin{array}{l}15 y \\
\text { female }\end{array}$ & $<\mathrm{p} 3$ & p75 & $\begin{array}{l}\text { MR, hydrocephalus with } \\
\text { shunt, corpus callosum } \\
\text { agenesis }\end{array}$ & $\begin{array}{l}\text { inguinal hernias, } \\
\text { bilateral conductive } \\
\text { hearing loss, ectopic } \\
\text { eruption of incisors, } \\
\text { occult spina bifida T2- } \\
\text { T3, scoliosis }\end{array}$ & $\begin{array}{l}\text { frontal bossing, synophrys, } \\
\text { prognathism }\end{array}$ & $\begin{array}{l}\text { yes } \\
\text { palmoplantar pits, } \\
\text { bifid ribs }\end{array}$ & $\begin{array}{l}\text { HR karyotype, } \\
\text { RLFP } \\
\text { polymorphisms }\end{array}$ & $\begin{array}{l}\text { 9q22q22 de } \\
\text { novo }\end{array}$ & paternal & $10-16 \mathrm{~cm}$ & deleleted & deleted \\
\hline [3, patient 2] & $\begin{array}{l}26 y \\
\text { female }\end{array}$ & short & microcephaly & MR & $\begin{array}{l}\text { PDA, CoA, anomalous } \\
\text { right subclavian artery, } \\
\text { bilat conductive } \\
\text { hearing loss }\end{array}$ & $\begin{array}{l}\text { frontal bossing, hypertelorism, } \\
\text { prognatism, prominent supraorbital } \\
\text { ridges, } \\
\text { high palate }\end{array}$ & $\begin{array}{l}\text { yes } \\
\text { multiple basal cell } \\
\text { carcinomas, } \\
\text { leiomyoma coli, } \\
\text { multiple bifid ribs } \\
\text { odontogenic } \\
\text { ceratocysts, } \\
\text { ameloblastoma }\end{array}$ & $\begin{array}{l}\text { HR karyotype, } \\
\text { RLFP } \\
\text { polymorphisms } \\
\text { FISH }\end{array}$ & $\begin{array}{l}\text { 9q22q32 de } \\
\text { novo }\end{array}$ & $\begin{array}{l}\text { maternal t(ins } \\
\text { [9] } \\
\text { [p22q32q22];16) } \\
\text { (p22;q21) mat }\end{array}$ & $22-39 \mathrm{~cm}$ & deleted & deleted \\
\hline [9] & $14 \mathrm{y}$ male & $<\mathrm{p} 3$ & $<\mathrm{p} 3$ & $\begin{array}{l}\text { severe MR } \\
\text { brachycephaly, dilated } \\
\text { ventricles }\end{array}$ & $\begin{array}{l}\text { undescended testes, } \\
\text { left pes equinovarus, } \\
\text { I partial I-IV toe } \\
\text { syndactyly, }\end{array}$ & $\begin{array}{l}\text { frontal bossing, hyperteloris, } \\
\text { epicanthi, } \\
\text { palpebral fissures slant down, broad } \\
\text { eyebrows, wide mouth, thick lips, } \\
\text { irregular dentition, } \\
\text { ears small, no ear lobes, } \\
\text { short neck, wide internipple distance, } \\
\text { hypoplastic genitalia, } \\
\text { tapering fingers, small toes, medial } \\
\text { deviation of toes }\end{array}$ & $\mathrm{nr}$ & karyotype & $\begin{array}{l}\text { 9q22q32 de } \\
\text { novo }\end{array}$ & maternal & $\mathrm{nr}$ & $\mathrm{nr}$ & $\mathrm{nr}$ \\
\hline [21] & $\begin{array}{l}15 \mathrm{~m} \\
\text { male }\end{array}$ & p25 & p75 & $\begin{array}{l}\text { severe MR, } \\
\text { partial aplasia of corpus } \\
\text { callosum, } \\
\text { dilatation of ventricles }\end{array}$ & $\begin{array}{l}\text { laryngomalacia, } \\
\text { cleft palate, } \\
\text { PDA, abnormal aortic } \\
\text { valve, } \\
\text { epiglottic dysplasia, } \\
\text { abnormal vocal cords }\end{array}$ & $\begin{array}{l}\text { frontal bossing, palpebral fissures slant } \\
\text { down, hypertrichosis, long eyelashes, } \\
\text { epicanthus inversus, } \\
\text { nose short, brodge depressed, nares } \\
\text { upturned, } \\
\text { long philtrum, } \\
\text { small mouth, thin upper lip, } \\
\text { receding chin, } \\
\text { ears large and low set, } \\
\text { large lobules, } \\
\text { loose skin on cheeks }\end{array}$ & $\mathrm{nr}$ & $\begin{array}{l}\text { karyotype (500 } \\
\text { bands) }\end{array}$ & $\begin{array}{l}\text { 9q22q2207 de } \\
\text { novo }\end{array}$ & & $\mathrm{nr}$ & $\mathrm{nr}$ & $\mathrm{nr}$ \\
\hline [2, patient 3] & $\begin{array}{l}7 \mathrm{~m} \\
\text { female }\end{array}$ & p90 & p75 & $\begin{array}{l}\text { mild to moderate MR, } \\
\text { brachycephaly, } \\
\text { hydrocephalus }\end{array}$ & $\begin{array}{l}\text { VSD, } \\
\text { PDA hallux valgus }\end{array}$ & $\begin{array}{l}\text { hypertelorism,epicanthi, } \\
\text { synophrys, } \\
\text { ptosis, } \\
\text { philtrum short ears posteriorly rotated }\end{array}$ & $\mathrm{nr}$ & HR karyotype & $\begin{array}{l}\text { 9q22.3q31.1 de } \\
\text { novo }\end{array}$ & $\begin{array}{l}\text { paternal dir ins } \\
(4 ; 9)(\text { (q33; } \\
\text { q22.3q31.1) pat }\end{array}$ & $\mathrm{nr}$ & $\mathrm{nr}$ & $\mathrm{nr}$ \\
\hline [35] & $\begin{array}{l}\text { infant } \\
\text { male }\end{array}$ & $\mathrm{nr}$ & $\mathrm{nr}$ & $\begin{array}{l}\text { asymmetric ventricles, } \\
\text { partial fusion of } \\
\text { cerebellar hemispheres, } \\
\text { polymicrogyria, delayed } \\
\text { cerebellar neuronal } \\
\text { migration, enlarged } \\
\text { massa intermedia }\end{array}$ & $\begin{array}{l}\text { cryptorchidism, focal } \\
\text { glomerulosclerosis, } \\
\text { accessory spleen, } \\
\text { partial fusion of } \\
\text { vertebrae D2 and D3, } \\
\text { pectus excavatum, } \\
\text { irregular ribs } \\
\text { interphalangeal } \\
\text { ankylosis }\end{array}$ & & $\mathrm{nr}$ & karyotype & $\begin{array}{l}\text { 9q22q32 de } \\
\text { novo }\end{array}$ & & $\mathrm{nr}$ & $\mathrm{nr}$ & $\mathrm{nr}$ \\
\hline
\end{tabular}


Table 1 Summary of clinical and molecular features of previously reported patients with constitutional 9q22 deletions and the present ones. (Continued)

\begin{tabular}{|c|c|c|c|c|c|c|c|c|c|c|c|c|c|}
\hline [10] & $3 \mathrm{~m}$ male & $\mathrm{nr}$ & $\mathrm{nr}$ & $\begin{array}{l}\text { death at } 3 \text { months, } \\
\text { seizures }\end{array}$ & $\begin{array}{l}\text { duodenal atresia, } \\
\text { malrotation, } \\
\text { Meckel diverticulum, } \\
\text { multilobulated spleen, } \\
\text { accessony spleens } \\
\text { renal dysplasia, } \\
\text { hydroureter } \\
\text { polydactyly of hand, } \\
\text { syndactyly of feet } \\
\text { angulated clavicles } \\
\text { thorax asymmetric }\end{array}$ & $\begin{array}{l}\text { palpebral fissures slant down, epicanthi, } \\
\text { hypotelorism, hirsutism, short palpebral, } \\
\text { fissures, depressed nasal bridge, } \\
\text { auditory canals narrow, } \\
\text { philtrum long }\end{array}$ & $\begin{array}{l}\text { nr } \\
\text { unusual ribs, }\end{array}$ & $\begin{array}{l}\text { karyotype, } \mathrm{Q}, \mathrm{C} \\
\text { and R bands }\end{array}$ & $\begin{array}{l}\text { 9q22q32 de } \\
\text { novo }\end{array}$ & maternal & $\mathrm{nr}$ & $\mathrm{nr}$ & $\mathrm{nr}$ \\
\hline$[1$, patient 1] & $14 \mathrm{y}$ male & $-1 \mathrm{SD}$ & OSD & $\begin{array}{l}\text { severe MR, } \\
\text { epilepsy }\end{array}$ & & $\begin{array}{l}\text { hypertelorism, } \\
\text { palpebral fissures slant down, nose } \\
\text { short, nares anteverted, } \\
\text { philtrum long, mild micrognathia }\end{array}$ & $\mathrm{nr}$ & $\begin{array}{l}\text { karyotype with } \\
\text { R bands }\end{array}$ & $\begin{array}{l}\text { 9q1 1q22 de } \\
\text { novo }\end{array}$ & & $\mathrm{nr}$ & $\mathrm{nr}$ & $\mathrm{nr}$ \\
\hline Present case 3 & 37 y male & $-1 S D$ & $-0,25 \mathrm{SD}$ & $\begin{array}{l}\text { normal cognition, } \\
\text { dysarthria }\end{array}$ & $\begin{array}{l}\text { grade IV } \\
\text { vesicoureteral reflux, } \\
\text { hydroureter and } \\
\text { hypoplastic left } \\
\text { kidney. funnel chest, } \\
\text { three lower-most } \\
\text { costal cartilages } \\
\text { broadly fused }\end{array}$ & $\begin{array}{l}\text { deepset and small toenails, palpebral } \\
\text { fissures slant down, high bridged nose, } \\
\text { narrow nares, long deep furrowed } \\
\text { philtrum, ears with broad helices and } \\
\text { uplifted lobuli, short } 2^{\text {nd }} \text { finger nails }\end{array}$ & no & $\mathrm{aCGH}$ & $\begin{array}{l}\text { 9q22.2q22.31 } \\
\text { de novo }\end{array}$ & & $5,3 \mathrm{Mb}$ & $\begin{array}{l}\text { not } \\
\text { deleted }\end{array}$ & deleted \\
\hline Present case 1 & $\begin{array}{l}8,5 y \\
\text { female }\end{array}$ & $0,1 S D$ & $+0,55 \mathrm{D}$ & $\begin{array}{l}\text { moderate MR, } \\
\text { dysarthria }\end{array}$ & & $\begin{array}{l}\text { short neck, slight ptosis on the right, } \\
\text { downward slant of the palpebral } \\
\text { fissures, narrow nose, small nares, long } \\
\text { philtrum with a narrow deep groove, } \\
\text { tented upper lip, ears with broad helices } \\
\text { and uplifted lobuli. toe nails II-V } \\
\text { bilaterally small }\end{array}$ & no & $\mathrm{aCGH}$ & $9 q 22.2 q 22.31$ & paternal & 5,3 Mb & $\begin{array}{l}\text { not } \\
\text { deleted }\end{array}$ & deleted \\
\hline Present case 2 & $\begin{array}{l}4 y \\
\text { female }\end{array}$ & $-0,25 \mathrm{D}$ & $+1 S D$ & $\begin{array}{l}\text { moderate MR, } \\
\text { dysarthria }\end{array}$ & & $\begin{array}{l}\text { down slanting palpebral fissures, mild } \\
\text { left side ptosis, narrow nares, uplifted } \\
\text { ear lobuli and thick helices, long } \\
\text { philtrum with a deep furrow, thin and } \\
\text { tented upper lip. }\end{array}$ & no & $\mathrm{aCGH}$ & 9q22.2q22.31 & paternal & $5,3 \mathrm{Mb}$ & $\begin{array}{l}\text { not } \\
\text { deleted }\end{array}$ & deleted \\
\hline
\end{tabular}

All values given as reported in each original paper.

Abbreviations: $\mathrm{nr}=$ no report, $\mathrm{y}=$ year, $\mathrm{m}=$ month, $\mathrm{OFC}=$ head circumference; $\mathrm{CNS}=$ central nervous system; $\mathrm{MR}=$ mental retardation; $\mathrm{p}=$ percentile; $\mathrm{SD}=$ standard deviation, $\mathrm{aCGH}=$ array comparative genomic hybridization, $\mathrm{HR}=$ high resolution. 
analysis was conducted on patient 1 according to standard protocols. Targeted FISH-analysis of lymphocyte cells of patient 3 was performed using BAC-probe RP11-30L4.

\section{Molecular karyotyping}

Mental retardation in addition to subtle but undisputable dysmorphic features in patients 1 and 2 were indications to perform additional high-resolution analysis by comparative genomic hybridization $(\mathrm{CGH})$ and single nucleotide polymorphism (SNP) arrays. Standard molecular karyotyping was performed using a 244 K CGH array (Agilent Technologies, Santa Clara, CA, USA), as previously described [11].

Array CGH results were confirmed by SNP array analysis of patients 1 and 3 and the paternal grandparents, using the Genome-Wide Human SNP array 6.0 according to manufacturer instructions (Affymetrix, Santa Clara, CA, USA). The SNP 6.0 array contains 906,000 SNP probes and 946,000 copy number probes and has an average resolution of $0.7 \mathrm{~kb}$. Data was extracted using the Genotyping console software V.3.0.2 with default settings, including the BRLMM-P-Plus algorithm and the Hidden-Markow Model for smoothing the copy number data. As a reference set we used data from 90 Caucasian HapMap samples. The extracted data was further analyzed using the Chromosome Analysis Suite software V.1.0. Copy number changes were called and filtered based on reference data and a minimum amount of 10 consecutive probes. All changes that were called were further compared to the Database of Genomic Variants (DGV, http://projects.tcag.ca/variation) as well as in-house data (unpublished material). All array data is stored in and available from the CanGEM Database (http://www.cangem.org). Array CGH and SNP data were analyzed using the reference genome build 18 (NCBI 36).

\section{Clinical report}

The family has four children and in addition the mother has a history of four first trimester miscarriages. Patients 1 and 2 are the $2^{\text {nd }}$ and $3^{\text {rd }}$ born children in the family. The psychomotor development of the eldest daughter has been within normal limits, but she suffers from short attention span and need special support at school. The youngest boy is considered healthy at 10 months of age. The paternal grandparents have neither learning problems nor congenital abnormalities and the family history is unremarkable (Figure 1).

Patient 1, a girl, was born after an uneventful pregnancy. She had normal birth size (3450 g, $49 \mathrm{~cm}$, OFC $35 \mathrm{~cm}$ ) and Apgar score was 10 at 1 and 5 minutes. A drop in blood hemoglobin from $172 \mathrm{~g} / \mathrm{l}$ to $127 \mathrm{~g} / \mathrm{l}$ was observed during the first day and she received a red cell transfusion. No evidence for bleeding, infection, hemoglobin abnormality, or immunization were found. She was discharged in good condition at the age of one week. She has suffered from recurrent middle ear infections since 1 year of age and repeated insertion of grommets and prophylactic antibiotics were not helpful. She
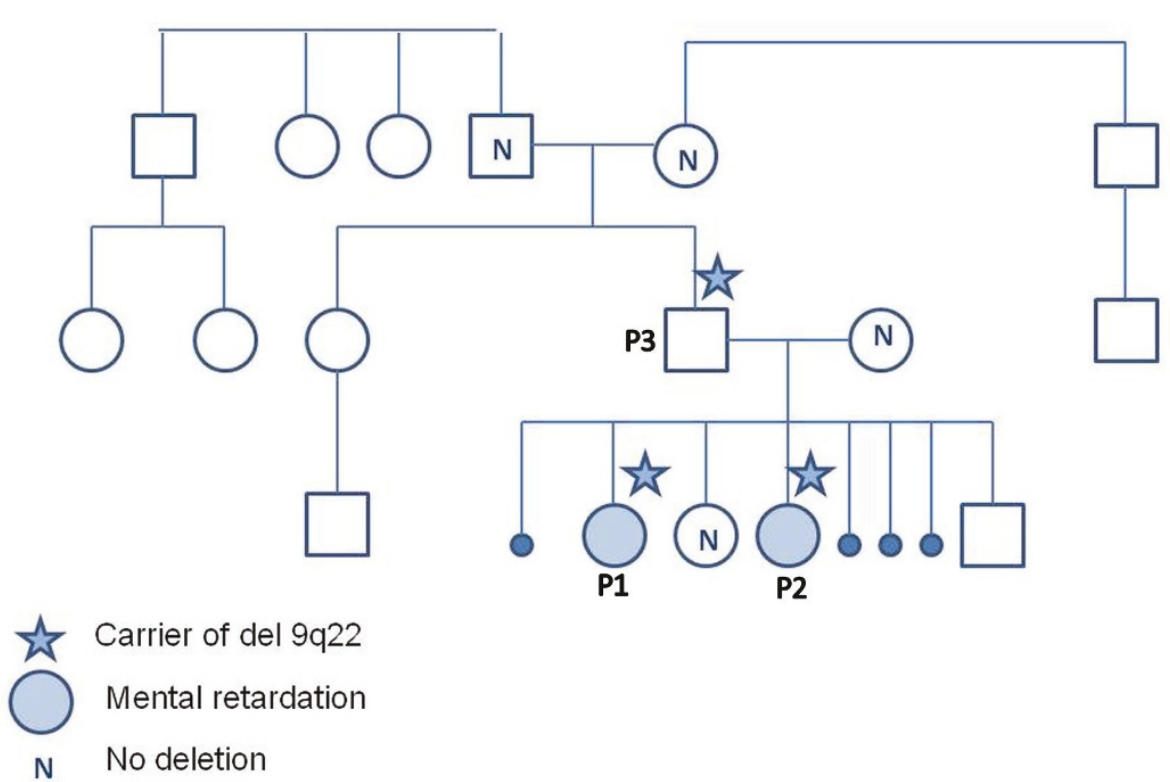

Figure 1 Pedigree of the family Patients 1, 2, and 3 all have the deletion of 9q22, as indicated by the star. The healthy mother, sister, and paternal grandparents, do not have the deletion, as indicated by $\mathrm{N}$. The healthy brother was not tested for the deletion. Only patients 1 and 2 (P1, P2) have mental retardation. 
developed bronchial asthma at the age of four years. At 2 and 3 years she had febrile convulsions but EEG recordings were normal.

She learned to walk independently at 20 months and spoke her first words at 15 months. At 4 years neuropsychological examination showed mild mental retardation, defective linguistic development and clumsy fine motor performance. At 9 years she attends a school for developmentally handicapped children and has learned elements of reading and writing. She needs help in basic daily skills. She speaks using sentences, but her speech is dysarthric. The vicious circle of ear infections continues. She needs treatment for asthma and uses melatonin for sleep problems.

During the first two years her height was at $+2 \mathrm{SD}$ and has thereafter approached +0.5SD (target height -0.5SD). She has overweight (BMI 24) since 2 years of age. The OFC grows steadily at $-0.5 \mathrm{SD}$. She has normal body proportions, short neck, slight ptosis on the right, downward slant of the palpebral fissures, narrow nose, small nares, long philtrum with a narrow deep groove, tented upper lip, ears with broad helices and uplifted lobuli. Her toe nails II-V are small on both feet; otherwise feet, toes, hands, fingers and fingernails are normal (Figures 2 and 3).

Blood lymphocyte counts have repeatedly been normal. She has normal immunoglobulin levels including IgG subclasses, $\mathrm{C} 3$ and $\mathrm{C} 4$ and alpha-1-antitrypsin and no IgE related allergies have been found. Urine metabolic screen was negative an no vacuolated lymphocytes were detected. The brain MRI and US of kidneys and abdominal organs were normal at the age of 4.5 years. $\mathrm{X}$-rays of the thorax showed no skeletal anomalies. Chromosome analysis (400 bands), subtelomere-FISH, 22q11 FISH (TUPLE1 probe), and FRAXA mutation analysis were normal.

Patient 2 is the younger sister of patient 1 . She was born at term after an uneventful pregnancy. The mother

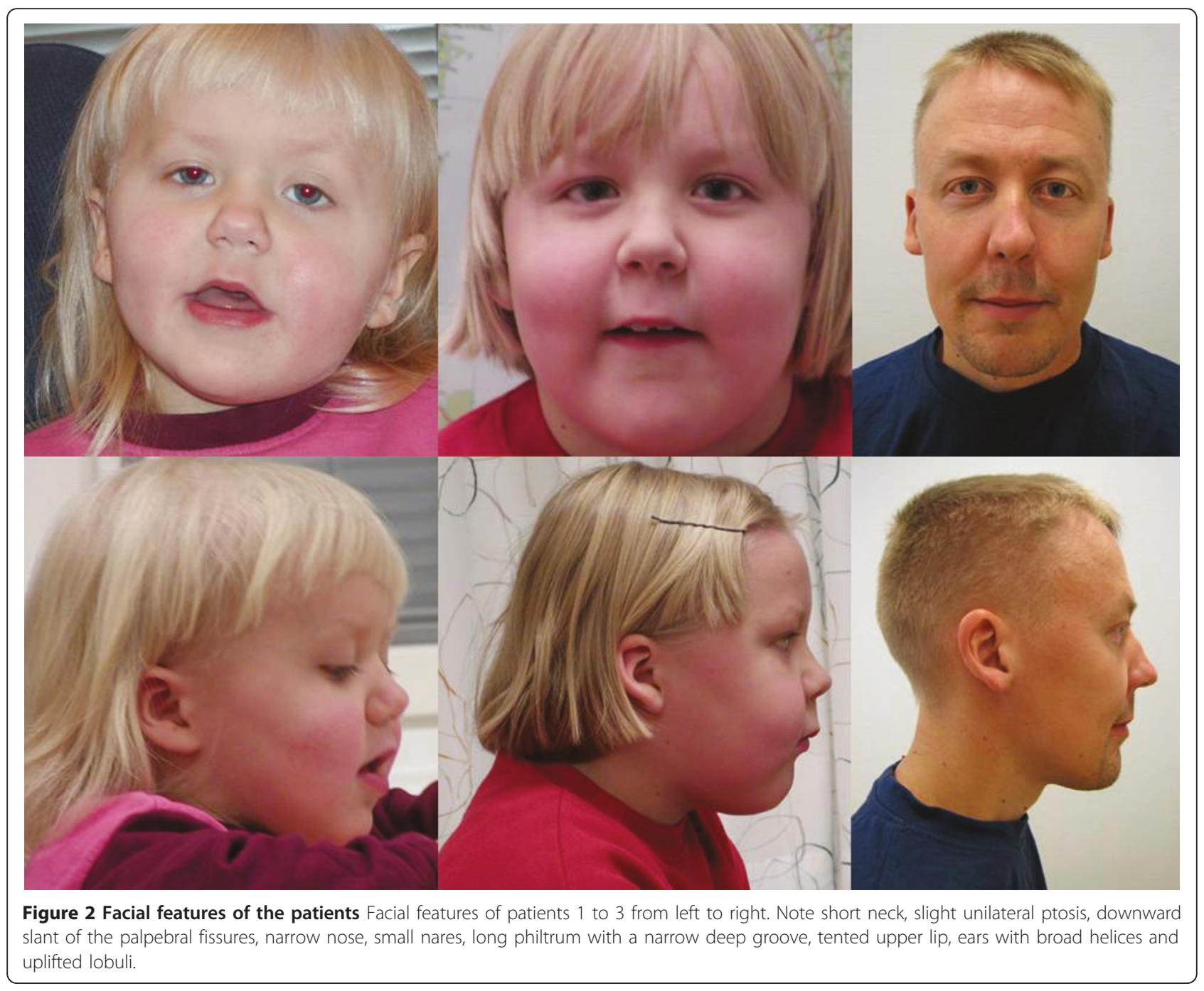




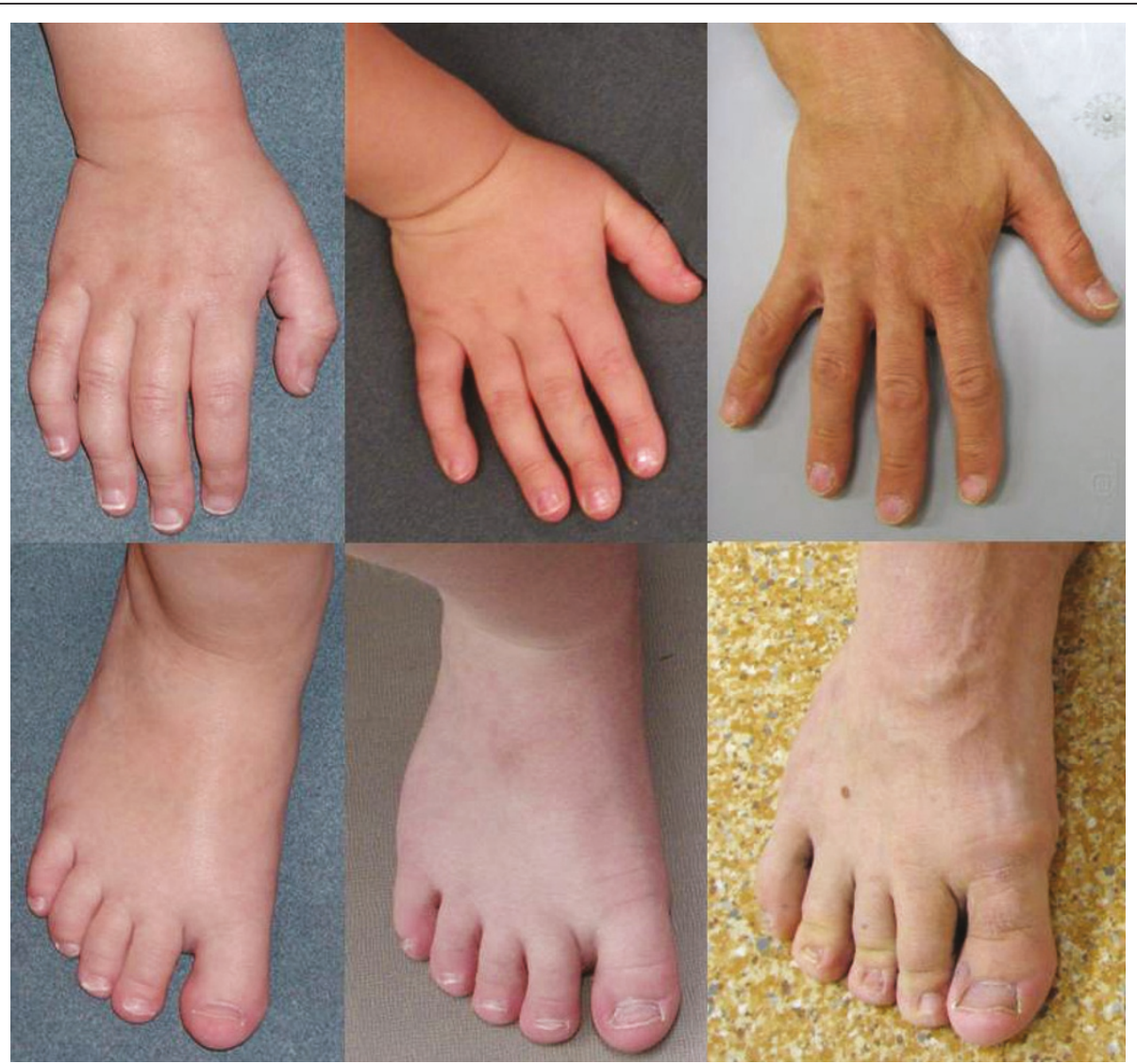

Figure 3 Hands and feet of the patients Hands and feet of patients 1 to 3 from left to right. Note that patient 1 has small toe nails II-V on both feet and patient 3 has small and deep-set toenails and his $2^{\text {nd }}$ finger nails are short.

was treated with acyclovir for herpes simplex type 1 infection from the $17^{\text {th }}$ week up until the delivery and with hydroxysitsin for her itching. Her birth size was normal ( $3770 \mathrm{~g}, 52 \mathrm{~cm}$, OFC $35 \mathrm{~cm}$ ) At the age of three weeks she was admitted to hospital for apneas caused by pulmonary and middle ear infections and needed mechanical ventilation for four days. The causative microorganism was not identified. EEG recording showed right sided spikes and phenobarbital treatment was started. At 6 months the medication was discontinued due to absence of epileptic symptoms and normal EEG. Brain MRI, cardiac conduction examinations, and cardiac ultrasound were normal.

Psychomotor developmental delay was obvious since the age of 6 months. She learned to walk independently at 2.5 years, spoke words at 3 years and short sentences at 5 years of age. At 5 years her speech is dysarthric, she has problems of falling asleep in the evening and treatment with melatonin has shown modest success. She has sudden attacks of aggression and abrupt standstills when she is withdrawn from contact and stares or cries and holds her head between her hands. EEG recordings show no epileptic activity. Her ability of reciprocal contact is very defective. She also suffers from abundant ear infections and from a tendency to asthmatic bronchitis. During the $1^{\text {st }}$ year her height increased from +1SD to +2SD and then has declined to -0,5SD (her target height). Her OFC has grown at OSD during the first 6 months of life and thereafter has remained at $+1 \mathrm{SD}$. She shows down slanting palpebral fissures, mild left side ptosis, narrow nares, uplifted ear lobuli and thick helices, long philtrum with a deep furrow, thin and tented upper lip (Figures 2 and 3). Her immunoglobulin levels are normal, but IgE related food allergy has been suspected.

Patient 3 is the father of patients 1 and 2. He was born term after a normal pregnancy. His birth size was normal $(53 \mathrm{~cm}, 4090 \mathrm{~g})$ and Apgar score was 9 at 1 and 10 at 5 minutes. At the age of 2.5 months he had a febrile pyelonephritis and urological examinations revealed grade IV vesicoureteral reflux, hydroureter and hypoplastic left kidney. In adult age his renal function has been normal. He suffered from recurrent middle ear infections and secretory otitis up to his teens. Surgery to correct his funnel chest was attempted when he was 6 years old. The surgery report describes the three lowermost costal cartilages being broadly fused. The 


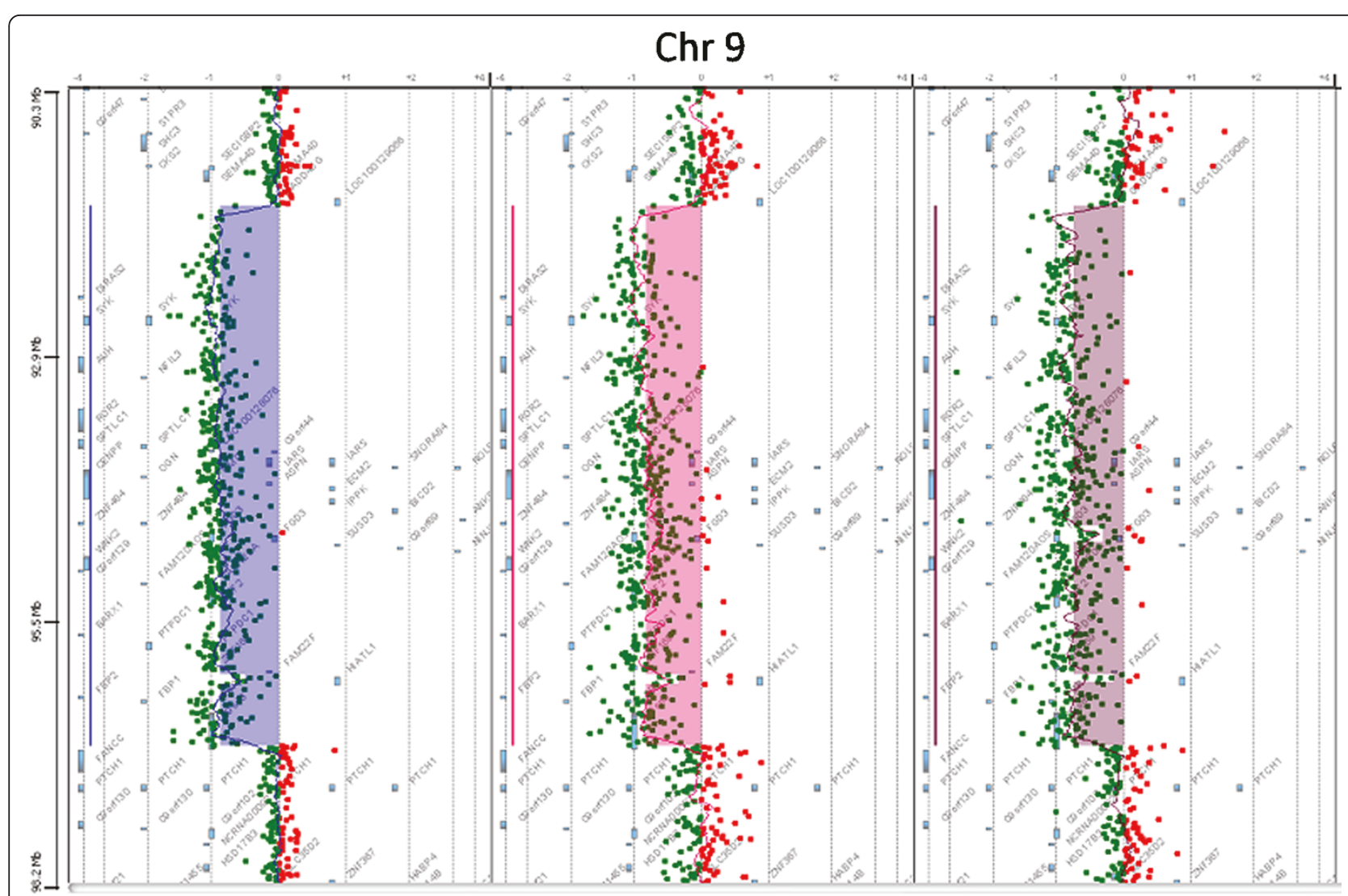

Figure 4 Array CGH results revealed a deletion at $\mathbf{9 q 2 2}$ in all three patients Array CGH results of patients 1,2 and 3 revealed a 5.3 Mb deletion in all patients at 9 q22.2q22.32. The shaded area indicates the deleted area with an average log2-ration of -1 , indicating loss of one copy of the genomic segment.

immediate postoperative X-ray could be traced and it showed normal claviculae, ribs and thoracic vertebrae.

His psychomotor development during childhood was considered normal except speech development and he had speech therapy up to his teens. At adult age he still suffers from dysarthria. His school and military service history are unremarkable. Presently he works as a mailman and studies to become a nurse's aide.

At 1 year of age his height was $80 \mathrm{~cm}(+1 \mathrm{SD})$. His adult height is $171,5 \mathrm{~cm}(-1 \mathrm{SD})$, weight $68 \mathrm{~kg}$ (BMI 23) and OFC $57 \mathrm{~cm}(-0,8 \mathrm{SD})$. He has partial upper denture after removal of decayed front teeth. The thoracic cage shows sequelae of childhood surgery and the sternum appears short but body-limb proportions are normal. He has down-slanting palpebral fissures, high nasal bridge, narrow nares, long philtrum with deep furrow, and the ears have broad helices and uplifted lobule. The 2nd finger nails are short and he has deep set toe nails. (Figures 2 and 3).

\section{Results}

Karyotype analysis of patients 1, 3, and the mother, were normal. Sub-telomere FISH analysis of patient 1 was also normal.
Both array CGH and SNP array analysis revealed an identical 5.3 Mb deletion of chromosome 9q22.2q22.32 in all three patients, ranging from basepair 91,523,558 to 96,858,929 (Agilent probes A_16P18688441-A_16_ P02135862), covering 30 genes (Figure 4). The average $\log 2$-ratio was -1 , indicating a one-copy deletion.

The parents of patient 3 and the older sister of patients 1 and 2 were studied for the microdeletion with normal results. Targeted FISH-analysis of at least 80 metaphase spreads, using BAC-probe RP11-30L4, was conducted in an effort to detect mosaicism 9q22 in patient 3. All metaphases showed the deletion of 9q22. Although these results do not exclude germline mosaicism of patient 3, the likelihood is small.

\section{Discussion}

The clinical and molecular findings of the previously reported 27 live patients and our 3 patients with constitutional 9q22 deletions are summarized in Table 1. Two prenatally terminated fetuses are not included in the analysis $[12,13]$. Based on available breakpoint information, at least eight reported patients have an overlapping deletion with the one detected in this family (Figure 5) [5,7,14-17]. 


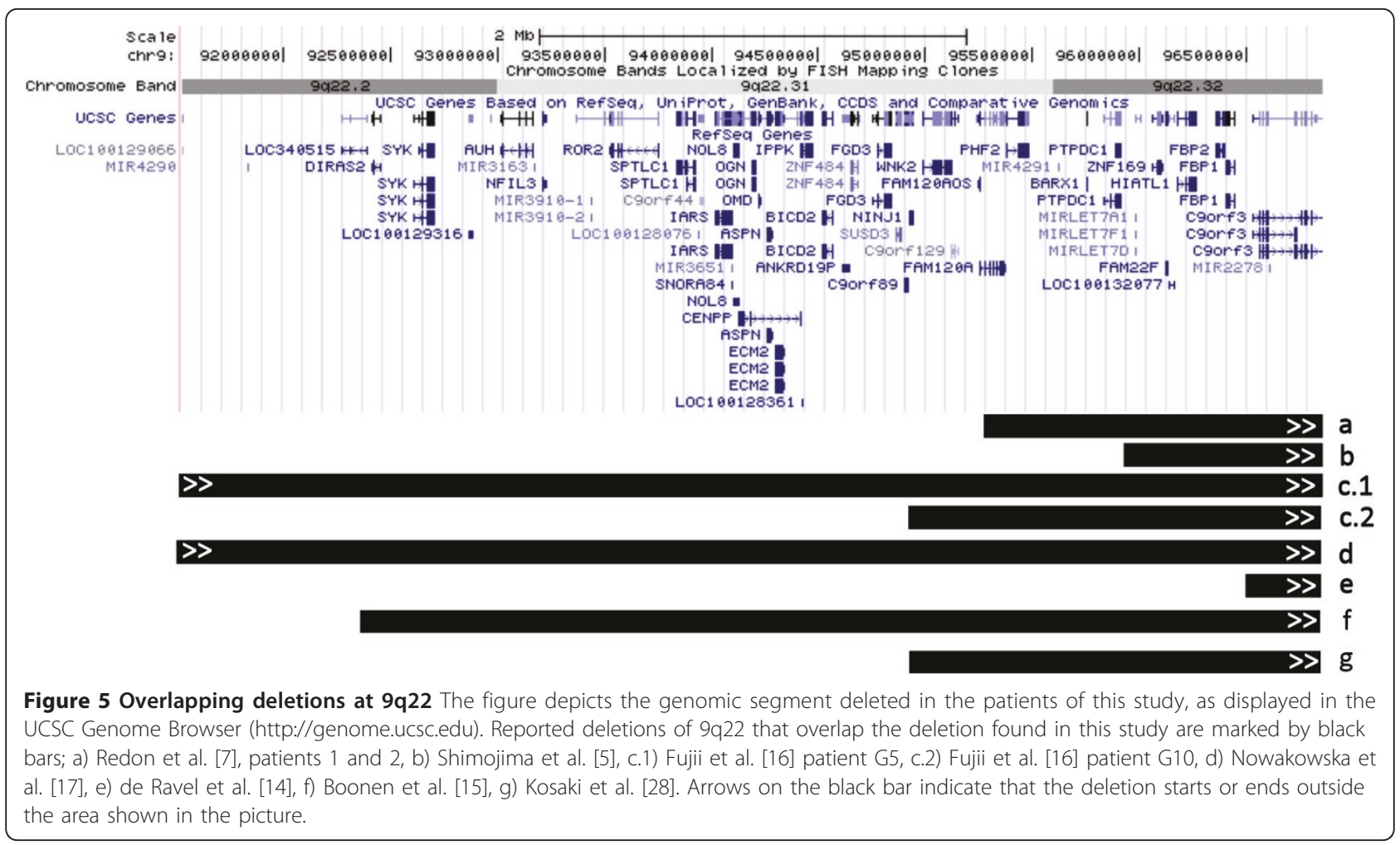

The deletion found in our three patients leaves the PTCH1 gene intact. Instead, according to DECIPHER database (the DECIPHER consortium, http://decipher. sanger.ac.uk/), three genes in the deleted area, viz. $S Y K$, IARS and ASPN, are scored as likely haploinsufficient [18]. $S Y K$ is involved in several important biological processes as discussed below. An allelic variant of ASPN has been implicated in osteoarthritis [19] and lumbar disc degeneration [20]. Neither of these features was present in our patients. IARS encodes an isoleucyl-tRNA synthetase protein and deletions of this gene have not been associated with human disease. In addition, three clustered micro-RNAs (miRNA) called hsa-let-7a, hsalet-7f and hsa-let-7d are found in the deleted area.. All of these miRNAs have a common primary target gene, HMGA2, as scored by Target Scan (http://www.targetscan.org/) and miRBase (http://www.mirbase.org/), indicating that they all function as repressors of this gene. The deletion is flanked by some short segmental duplications. These, however, do not lie at the breakpoints but further up-/downstream and thus it is unclear whether the rearrangement could initially have been due to non-allelic homologous recombination (NAHR).

Almost all patients published so far have been reported to have dysmorphic facial features. However, no unique facial gestalt does emerge. All three patients described here had similar mild dysmorphic features: downward slanting palpebral fissures, high bridged narrow nose with small nares, deep grooved long philtrum, tented and thin upper lip, ears with broad helices and uplifted lobuli. In addition patients 1 and 3 have small toenails. When compared with the previous reports, only the patient reported by Olivieri et al. (2003) has facial features closely resembling those of our patients [8].

A significant common feature in our three patients is incessant middle ear and upper respiratory tract infections. No major immunological deficiency, however, was found to cause the vicious circle of infections, and in the father this symptom disappeared at his teens. Only three previously reported patients suffered from recurrent respiratory infections $[14,15,21]$. Interestingly, the region deleted in our patients includes the gene Spleen Tyrosine Kinase (SYK) (MIM "600085) which is widely expressed in hematopoietic cells and other cells of epithelial origin. $S Y K$ plays an important role in regulation of innate immunity and inflammatory response, and is involved in several human diseases such as allergy, autoimmunity, and haematological malignancies (reviewed by Mócsai et.al [22]). The infections seen in our patients could be linked to reduced $S Y K$ activity and thereby reduced, albeit not lacking, ability to activate the inflammatory response. Recent studies in mice show that a $S Y K$-deletion reduces their antibacterial host defence [23]. SYK is deleted in the patients with recurrent respiratory infections reported by Pfeiffer et al., and 
Boonen et al. but not de Ravel et al. [14,15,21]. The relevance of its haploinsufficiency to human recurrent early-age upper airway infections might be a subject for further studies.

Mental retardation among the reported 9q22 deletion patients is common and usually moderate to severe. Only the 4-year-old patient of Shimojima et al. with a 2,3 $\mathrm{Mb}$ deletion is reported to have normal development [5]. The intelligence of the father in our family is within normal limits although a formal neuropsychological testing has not been performed. Instead, his two daughters carrying a similar deletion are moderately retarded. In genetic counseling a similar deletion found both in patients with mental retardation and in a family member with normal cognitive function understandably poses difficulties. The cause of the variable expression remains to be found. According to the Database of Genomic Variants (http://projects.tcag.ca/variation) the deletion described here has not been found in healthy controls. Reduced penetrance was not found to be due to mosaicism admitting the fact that in addition to lymphocytes no other tissue like semen or buccal cells were examined. The parents did not consider further studies for mosaicism important and thus it was not pursued.

Another explanation for variable expression would be two simultaneous genomic hits acting either independently or by affecting the same signaling pathway [24]. In our family the first hit would be the deletion, but the second hit would need other methods to be detected. The paternal microdeletion could also reveal a maternal allelic recessive defect and the consequent autosomal compound heterozygosity in the two daughters would be the cause of their cognitive impairment. No obvious candidate gene is situated in the deleted region. Microdeletions are increasingly frequently encountered associated with the phenomenon of variable penetrance and even nonpenetrance, $15 \mathrm{q} 13.3$ microdeletion syndrome being one example [25]. The deleted region is not known to be affected by imprinting that could cause cognitive differences among the patients.

A common developmental problem of our three patients is dysarthria, which is severe and making even the speech of the cognitively normal father difficult to comprehend. A similar problem has not been described in the previously published patients and further studies are needed to explore whether a locus for dysarthria resides in the region defined by the deletion.

Both daughters in our family had symptoms suspected to be epileptic although the diagnosis could not be confirmed. Eight patients from the literature suffer from epilepsy, but no characteristic seizure type emerged [1, 7, 10, 26 (two patients), 16 (two patients), 27].

Patient 3 had unilateral hypoplastic kidney and vesicoureteral reflux. Also three previous patients had a renal problem; hydronephrosis, renal dysplasia or hydroureter $[10,16,28]$. Interestingly, patient 3 had a congenital chest deformity similar to one of the diagnostic criteria of NBCCS [29]. Additional X-rays could have clarified the presence of possible other NBCCS related skeletal signs. The 9q22 deletion detected did not, however, include PTCH1 gene and since our patient otherwise had no indications for further X-ray examinations, it was considered clinically inappropriate to take them. The patient's chest deformity can be independent of the deletion since patients 1 and 2 do not have thoracic anomalies. Yet, one is tempted to speculate that it is a sign that the deletion might effect the expression of PTCH1.

Another clinically important gene included in the deletion is ROR2. Gain of function mutations of one allele are known to cause brachydactyly type B1 while a mutation of both alleles cause Robinow Syndrome [30,31]. However, haploinsufficiency of ROR2 has no effect on phenotype as shown by Oldridge et al., who described two unrelated patients with de novo deletions at 9 q22 [30]. Accordingly, none of the six previous patients, nor our patients, whose deletion includes ROR2, have either brachydactyly type $\mathrm{B} 1$ or features of Robinow Syndrome [[3]3 (restudied in 15), [8,15-17]] (Table 1).

Shimojima et al. suggested that del 9q22 might be a novel overgrowth syndrome by paternal imprinting [5]. Among the previous patients eight carry a deletion in the paternally inherited chromosome [[3] (patient 1), [5,7] (patients 1 and 2), [8,28]] and three are maternally deleted [[3] (patient 2), [9,10]]. These small numbers are naturally liable to bias. Indeed, the paternally deleted patients seem to have a large neonatal size. Among those with data on postnatal growth $3 / 5$ are mildly tall and $4 / 6$ have macrocephaly. Growth data of the three maternally deleted patients is scanty but none of them seems to be tall or to have macrocephaly. The maternal deletions reported appear to be much larger which implies a more significant genomic imbalance and could alone explain differences in phenotype (Table 1).

Recently Kosaki et al. [28] narrowed the region of the proposed overgrowth factor closer to PTCH1 gene by their patient's deletion in relation to that of the patients published by Shimojima et al. and Redon et al. $[5,7]$. Our family's deletion helps to bring it even closer to PTCH1. On the other hand tallness and macrocephaly are also inherent features of NBCCS [32]. In a thorough survey of a large series of NBCCS patients Kimonis et al. observed no imprinting effect regarding any feature of the syndrome including macrocephaly [29]. Overgrowth like macrocephaly observed in del 9q22 patients could thus simply reflect the simultaneous haploinsufficiency of PTCH1. 
Our family underlines the importance to try and include both parental samples in array molecular karyotyping. In a more usual situation with only one developmentally retarded child in the family one might for several reasons be tempted to accept the analysis of the child alone or with only one parent and unknowingly thus get misleading information of the situation. Microdeletion $9 \mathrm{q} 22$ adds to the increasing number genomic imbalances that challenge genetic counseling.

\section{Conclusions}

In conclusion, 9q22 deletions are rare and both phenotypically and molecularly unique. In the majority the deletion contains the PTCH1 gene, which signifies that the patients in addition to the common mental retardation also develop the tumor proneness syndrome of Gorlin, which is to be taken into account in counseling and follow-up. We present the first familial 9q22 deletion, a father and his two developmentally delayed daughters. Their deletion leaves the PTCH1 gene intact. The father does not have significant cognitive problems but has renal and thoracic cage malformations while the daughters do not have congenital malformations. Dysarthric speech and prolonged tendency to ear and upper respiratory infections are common to all three. Major differences in psychomotor development warrant cautiousness in genetic counseling in patients with similar deletions.

\section{Consent}

Written informed consent was obtained from the father, the mother and the paternal grandparents for publication of this case report and accompanying images. A copy of the written consent is available for review by the Editor-in-Chief of this journal.

\section{Acknowledgements}

The authors thank the family for participating in this study. Dr. Eino Marttinen from the Department of Medical Imaging of the Helsinki University Hospital is acknowledged for important discussions about the radiological findings in patient 3. The Department of Medical Genetics, The Family Federation of Finland, is funded by Finland's Slot Machine Association (RAY). LS was funded by the Rinnekoti Research Foundation, Medicinska Understödsföreningen Liv och Hälsa r.f., and the State Appropriations of the Helsinki University Central Hospital.

\section{Author details \\ 'Department of Pathology, Haartman Institute and HUSLAB, University of Helsinki and Helsinki University Central Hospital, Haartmaninkatu 3, 00014 Helsinki, Finland. ${ }^{2}$ Department of Medical Genetics, The Family Federation of Finland, Helsinki, Finland. ${ }^{3}$ Institute for Integrated Medical Sciences, Tokyo Women's Medical University, Tokyo, Japan. ${ }^{4}$ Department of Medical Genetics, Turku University Hospital, Turku, Finland.}

\section{Authors' contributions}

LS carried out the molecular genetic studies, participated in the design of the study and drafted the manuscript. MP carried out the clinical studies, participated in the design of the study and drafted the manuscript. MS carried out the sample collection. TM composed the photos for publication.
TY and KS performed the FISH analysis and helped to draft the manuscript. $\mathrm{J}$ critically revised the manuscript. SK participated in the study design and coordination and critically revised the manuscript. All authors read and approved the final manuscript.

\section{Competing interests}

The authors declare that they have no competing interests.

Received: 16 December 2010 Accepted: 22 June 2011

Published: 22 June 2011

\section{References}

1. Turleau C, de Grouchy J, Chabrolle JP: Intercalary deletions of 9q. Ann Genet 1978, 21:234-236.

2. Farrell SA, Siegel-Bartelt J, Teshima I: Patients with deletions of 9q22q34 do not define a syndrome: three case reports and a literature review. Clin Genet 1991, 40:207-214.

3. Shimkets $R$, Gailani MR, Siu VM, Yang-Feng T, Pressman CL, Levanat $S$, Goldstein A, Dean M, Bale AE: Molecular analysis of chromosome $9 q$ deletions in two Gorlin syndrome patients. Am J Hum Genet 1996, 59:417-422.

4. Midro AT, Panasiuk B, Tumer Z, Stankiewicz P, Silahtaroglu A, Lupski JR, Zemanova Z, Stasiewicz-Jarocka B, Hubert E, Tarasow E, Famulski W, Zadrozna-Tolwinska B, Wasilewska E, Kirchhoff M, Kalscheuer V, Michalova K, Tommerup N: Interstitial deletion 9q22.32-q33.2 associated with additional familial translocation $\mathrm{t}(9 ; 17)(\mathrm{q} 34.11 ; \mathrm{p} 11.2)$ in a patient with Gorlin-Goltz syndrome and features of Nail-Patella syndrome. Am J Med Genet A 2004, 124A:179-191.

5. Shimojima K, Adachi M, Tanaka M, Tanaka Y, Kurosawa K, Yamamoto T: Clinical features of microdeletion 9q22.3 (pat). Clin Genet 2009, 75:384-393.

6. Cajaiba MM, Bale AE, Alvarez-Franco M, McNamara J, Reyes-Mugica M: Rhabdomyosarcoma, Wilms tumor, and deletion of the patched gene in Gorlin syndrome. Nat Clin Pract Oncol 2006, 3:575-580

7. Redon R, Baujat G, Sanlaville D, Le Merrer M, Vekemans M, Munnich A Carter NP, Cormier-Daire V, Colleaux L: Interstitial 9q22.3 microdeletion: clinical and molecular characterisation of a newly recognised overgrowth syndrome. Eur J Hum Genet 2006, 14:759-767.

8. Olivieri C, Maraschio P, Caselli D, Martini C, Beluffi G, Maserati E, Danesino C: Interstitial deletion of chromosome 9, int del(9)(9q22.31-q31.2), including the genes causing multiple basal cell nevus syndrome and Robinow/brachydactyly 1 syndrome. Eur J Pediatr 2003, 162:100-103.

9. Kroes HY, Tuerlings JH, Hordijk R, Folkers NR, ten Kate LP: Another patient with an interstitial deletion of chromosome 9: case report and a review of six cases with del(9)(q22q32). J Med Genet 1994, 31:156-158.

10. Ying KL, Curry CJ, Rajani KB, Kassel SH, Sparkes RS: De novo interstitial deletion in the long arm of chromosome 9: a new chromosome syndrome. J Med Genet 1982, 19:68-70.

11. Siggberg L, Ala-Mello S, Jaakkola E, Kuusinen E, Schuit R, Kohlhase J, Böhm D, Ignatius J, Knuutila S: Array CGH in molecular diagnosis of mental retardation - a study of 150 patients. Am J Med Genet A 2010, 152A:1398-1410.

12. Paoloni-Giacobino A, Floris E, Dahoun SP: Fetus with a 9q22q34 interstitial deletion and hygroma. Prenat Diagn 2000, 20:855-856.

13. L'Hermine AC, Aboura A, Simon-Bouy B, Robin F, Audibert F, Strouk N, Capron F, Frydman R, Tachdjian G: Female pseudohermaphroditism in a fetus with a deletion 9(q22.2q31.1). Prenat Diagn 2002, 22:652-655.

14. de Ravel TJ, Ameye L, Ballon K, Borghgraef M, Vermeesch JR, Devriendt K: Early detection of chromosome $9 \mathrm{q} 22.32 \mathrm{q} 31.1$ microdeletion and the nevoid basal cell carcinoma syndrome. Eur J Med Genet 2009, 52:145-147.

15. Boonen SE, Stahl D, Kreiborg S, Rosenberg T, Kalscheuer V, Larsen LA, Tommerup N, Brondum-Nielsen K, Tumer Z: Delineation of an interstitial 9q22 deletion in basal cell nevus syndrome. Am J Med Genet A 2005, 132A:324-328.

16. Fujii K, Ishikawa S, Uchikawa H, Komura D, Shapero MH, Shen F, Hung J, Arai H, Tanaka Y, Sasaki K, Kohno Y, Yamada M, Jones KW, Aburatani H, Miyashita T: High-density oligonucleotide array with sub-kilobase resolution reveals breakpoint information of submicroscopic deletions in nevoid basal cell carcinoma syndrome. Hum Genet 2007, 122:459-466.

17. Nowakowska B, Kutkowska-Kazmierczak A, Stankiewicz P, Bocian E, Obersztyn E, Ou Z, Cheung SW, Cai WW: A girl with deletion 9q22.1- 
q22.32 including the PTCH and ROR2 genes identified by genome-wide array-CGH. Am J Med Genet A 2007, 143A:1885-1889.

18. Huang N, Lee I, Marcotte EM, Hurles ME: Characterising and predicting haploinsufficiency in the human genome. PLoS Genet 2010, 6:e1001154.

19. Song JH, Lee HS, Kim CJ, Cho YG, Park YG, Nam SW, Lee JY, Park WS: Aspartic acid repeat polymorphism of the asporin gene with susceptibility to osteoarthritis of the knee in a Korean population. Knee 2008, 15:191-195.

20. Song YQ Cheung KM, Ho DW, Poon SC, Chiba K, Kawaguchi Y, Hirose $Y$, Alini M, Grad S, Yee AF, Leong JC, Luk KD, Yip SP, Karppinen J, Cheah KS, Sham P, Ikegawa S, Chan D: Association of the asporin D14 allele with lumbar-disc degeneration in Asians. Am J Hum Genet 2008, 82:744-747.

21. Pfeiffer RA, Lachmann E, Schreyer W, Volleth M: Deletion of 9q22: a new observation suggesting a specific phenotype. Ann Genet 1993, 36:167-170.

22. Mocsai A, Ruland J, Tybulewicz VL: The SYK tyrosine kinase: a crucial player in diverse biological functions. Nat Rev Immunol 2010, 10:387-402.

23. Van Ziffle JA, Lowell CA: Neutrophil-specific deletion of Syk kinase results in reduced host defense to bacterial infection. Blood 2009, 114:4871-4882.

24. Girirajan S, Rosenfeld JA, Cooper GM, Antonacci F, Siswara P, Itsara A, Vives L, Walsh T, McCarthy SE, Baker C, Mefford HC, Kidd JM, Browning SR, Browning BL, Dickel DE, Levy DL, Ballif BC, Platky K, Farber DM, Gowans GC, Wetherbee JJ, Asamoah A, Weaver DD, Mark PR, Dickerson J, Garg BP, Ellingwood SA, Smith R, Banks VC, Smith W, McDonald MT, Hoo JJ, French BN, Hudson C, Johnson JP, Ozmore JR, Moeschler JB, Surti U, Escobar LF, El-Khechen D, Gorski JL, Kussmann J, Salbert B, Lacassie Y, Biser A, McDonald-McGinn DM, Zackai EH, Deardorff MA, Shaikh TH, Haan E, Friend KL, Fichera M, Romano C, Gecz J, DeLisi LE, Sebat J, King MC, Shaffer $L G$, Eichler EE: A recurrent 16p12.1 microdeletion supports a twohit model for severe developmental delay. Nat Genet 2010, 42:203-209.

25. Masurel-Paulet A, Andrieux J, Callier P, Cuisset JM, Le Caignec C, Holder M, Thauvin-Robinet C, Doray B, Flori E, Alex-Cordier MP, Beri M, Boute O, Delobel B, Dieux A, Vallee L, Jaillard S, Odent S, Isidor B, Beneteau C, Vigneron J, Bilan F, Gilbert-Dussardier B, Dubourg C, Labalme A, Bidon C, Gautier A, Pernes P, Pinoit JM, Huet F, Mugneret F, Aral B, Jonveaux P, Sanlaville D, Faivre L: Delineation of 15q13.3 microdeletions. Clin Genet 2010, 78:149-161.

26. Yamamoto K, Yoshihashi H, Furuya N, Adachi M, Ito S, Tanaka Y, Masuno M, Chiyo H, Kurosawa K: Further delineation of 9q22 deletion syndrome associated with basal cell nevus (Gorlin) syndrome: report of two cases and review of the literature. Congenit Anom (kyoto) 2009, 49:8-14.

27. Chen CP, Lin SP, Wang TH, Chen YJ, Chen M, Wang W: Perinatal findings and molecular cytogenetic analyses of de novo interstitial deletion of $9 q$ (9q22.3->q31.3) associated with Gorlin syndrome. Prenat Diagn 2006, 26:725-729.

28. Kosaki R, Fujita H, Ueoka K, Torii C, Kosaki K: Overgrowth of prenatal onset associated with submicroscopic 9q22.3 deletion. Am J Med Genet A 2011, 155:903-905.

29. Kimonis VE, Goldstein AM, Pastakia B, Yang ML, Kase R, DiGiovanna JJ, Bale AE, Bale SJ: Clinical manifestations in 105 persons with nevoid basal cell carcinoma syndrome. Am J Med Genet 1997, 69:299-308.

30. Oldridge M, Fortuna AM, Maringa M, Propping P, Mansour S, Pollitt C, DeChiara TM, Kimble RB, Valenzuela DM, Yancopoulos GD, Wilkie AO: Dominant mutations in ROR2, encoding an orphan receptor tyrosine kinase, cause brachydactyly type B. Nat Genet 2000, 24:275-278.

31. Schwarzer W, Witte F, Rajab A, Mundlos S, Stricker S: A gradient of ROR2 protein stability and membrane localization confers brachydactyly type B or Robinow syndrome phenotypes. Hum Mol Genet 2009, 18:4013-4021

32. Haniffa MA, Leech SN, Lynch SA, Simpson NB: NBCCS secondary to an interstitial chromosome 9q deletion. Clin Exp Dermatol 2004, 29:542-544.

33. Musani V, Cretnik M, Situm M, Basta-Juzbasic A, Levanat S: Gorlin syndrome patient with large deletion in 9q22.32-q22.33 detected by quantitative multiplex fluorescent PCR. Dermatology 2009, 219:111-118.

34. Sasaki K, Yoshimoto T, Nakao T, Minagawa K, Takahashi Y, Watanabe Y, Tanabe C: A nevoid basal cell carcinoma syndrome with chromosomal aberration. No To Hattatsu 2000, 32:49-55.

35. Robb LJ, Vekemans M, Richter A, Meagher-Villemure K, Neilsen K, Der Kaloustian VM: Interstitial deletion of the long arm of chromsome 9 [abstract]. Am J Hum Genet 1991, 49(suppl):274.

doi:10.1186/1750-1172-6-45

Cite this article as: Siggberg et al:: 9q22 Deletion - First Familial Case. Orphanet Journal of Rare Diseases 2011 6:45

\section{Submit your next manuscript to BioMed Central and take full advantage of:}

- Convenient online submission

- Thorough peer review

- No space constraints or color figure charges

- Immediate publication on acceptance

- Inclusion in PubMed, CAS, Scopus and Google Scholar

- Research which is freely available for redistribution

Submit your manuscript at www.biomedcentral.com/submit 